\title{
Effect of precise partial scab removal on the repair of deep partial-thickness burn wounds in children: a retrospective study
}

\author{
Yunfei Chi", Huinan Yin", Xin Chen, Quan Hu, Wei Liu, Li Feng, Jiake Chai \\ Burn Institute, The Fourth Medical Center of the PLA General Hospital, Beijing, China \\ Contributions: (I) Conception and design: Y Chi, J Chai; (II) Administrative support: H Yin; (III) Provision of study materials or patients: X Chen; \\ (IV) Collection and assembly of data: Q Hu, W Liu, L Feng; (V) Data analysis and interpretation: Y Chi, J Chai, H Yin; (VI) Manuscript writing: All \\ authors; (VII) Final approval of manuscript: All authors. \\ \#These authors contributed equally to this work and should be considered as co-first author. \\ Correspondence to: Li Feng; Jiake Chai. Burn Institute, The Fourth Medical Center of the PLA General Hospital, 51 Fu Cheng Road, Beijing 100048, \\ China. Email: lifeng5586@163.com; cjk304@126.com.
}

Background: The choice of treatment methods for children with deep partial-thickness burn wounds (DPBWs) is an issue that requires careful consideration from surgeons. The purpose of this study was to evaluate the efficacy of precise partial scab removal (PPSR) in the treatment of DPBWs in children.

Methods: We retrospectively analyzed the clinical data of 78 children with DPBWs. The children were divided into a PPSR group ( $n=37)$ and a routine dressing change (RDC) group ( $=41)$. In the PPSR group, an electric dermatome was used to cut the scab in the early post-injury period. The thickness scale of the electric dermatome was set to $0.1 \mathrm{~mm}$. The scab was removed to the base with scattered bleeding points. There was still a small amount of necrotic tissue in the base of the wound. For the acellular dermal matrix, the first dressing change was about 1 week after surgery. The RDC group was given conventional wounddressing treatment. The wound dressing was changed with epidermal growth factor, silver-zinc antibacterial cream, and dressing change. The frequency of dressing change was adjusted once a day or once every other day depending on the condition of wound secretions. The hospitalization time, wound-healing time, fever duration, antibiotic use time, number of subsequent operations, and overall hospitalization expenses were compared between the two groups.

Results: The wound-healing time of the PPSR group was $19.86 \pm 6.4$ days, and the wound-healing time of the RDC group was $24.15 \pm 7.12$ days $(\mathrm{P}=0.0068)$. The duration of fever in the PPSR group and RDC group was $2.62 \pm 1.99$ and $4.44 \pm 3.10$ days, respectively $(\mathrm{P}=0.0032)$. The antibiotic use time in the PPSR group and RDC group was $4.0 \pm 1.33$ and $4.83 \pm 1.88$ days, respectively $(\mathrm{P}=0.0292)$. The overall hospitalization cost of the PPSR group and RDC group was yuan renminbi $¥ 37,852.84 \pm 10,894.64$ and $¥ 38,047.46 \pm 19,450.37$, respectively $(\mathrm{P}=0.9573)$.

Conclusions: PPSR can shorten wound-healing time, reduce the frequency of dressing changes, shorten the time of fever in children, lower the frequency of antibiotic use, and decrease number of dressing changes on the wound.

Keywords: Burns; children; scabs; deep partial-thickness burn wounds (DPBWs)

Submitted Aug 23, 2021. Accepted for publication Nov 16, 2021.

doi: $10.21037 /$ tp-21-500

View this article at: https://dx.doi.org/10.21037/tp-21-500 


\section{Introduction}

The choice of treatment methods for children with deep second-degree burns and scalds is a common concern for surgeons, who are required to approach this issue carefully (1). In children with heat burns, a wound area of more than $10 \%$ will often cause systemic symptoms, such as fever and decreased appetite, and may even develop into sepsis (2). Continuous fever may cause other negative effects for children who are still in the developmental stage. The connective tissue of children's skin is rich in matrix, and the water content of skin tissue is also higher than that of adults. Acute inflammatory edema is more likely to occur after deep second-degree burns. If a child's second-degree burn is treated late or improperly treated, it is very easy to become a $\mathrm{III}^{\circ}$ burn due to infection and other reasons. For children with deep second-degree wounds, a simple change of the wound may lead to a longer healing time, the burned necrotic tissue may stay on the wound for an extended period, and systemic symptoms may be severe and enduring, increasing the incidence of complications. Frequent dressing changes on the wound surface may also cause tremendous pain to children (3). In addition, we know that healing time is closely related to the formation of scars. A long healing time will lead to severe scar contractures, which will then affect the development of children to varying degrees (4). Therefore, choosing a treatment method of debridement and scab autologous skin transplantation for children with deep second-degree wounds may shorten the healing time and the duration of systemic symptoms but may also increase the scars in the skin graft area and the scars in the skin removal area. In recent years, the clinical application of escharotomy has been frequently reported. Escharotomy mainly involves removing part of the necrotic tissue as much as possible while preserving healthy tissue and can positively impact wound healing $(5,6)$. This method has been reported to have achieved good results in the treatment of deep second-degree burns in adults and children. Inspired by this method, we carried out partial scab removal with precise control of the depth. Precise partial scab removal (PPSR) uses an electric dermatome to control the depth of the partial scab removal. After the operation, acellular xenogeneic pig skin is used for reference (7). Our previous study proved that PPSR in early stage was effective and safe in a small sample (8). The purpose of this study was to further retrospectively analyze the efficacy and safety of PPSR in the treatment of children with deep seconddegree burns and scalds in a larger sample. We present the following article in accordance with the STROBE reporting checklist (available at https://dx.doi.org/10.21037/tp-21500).

\section{Methods}

\section{Research participants}

A total of 78 children with deep partial-thickness burn wounds (DPBWs) who were admitted to the Burn Department of the Fourth Medical Center of the PLA General Hospital from July 2017 to July 2020 were retrospectively included. Further inclusion criteria for the study were the following: (I) the DPBW was located on the extremities and trunk; (II) hospitalization occurred within 48 hours after injury; (III) the wound was clean without serious infection; (IV) there was no shock, lethargy, irritability, or obvious thirst before the operation, and the extremities were warm, with a urine output of $\geq 1 \mathrm{~mL} / \mathrm{kg} / \mathrm{h}$ and a heart rate of $\leq 140 / \mathrm{min}$; (V) ages between 6 months and 6 years old; (VI) overall burn area of $10-40 \%$, and a DPBW area of $>5 \%$. The exclusion criteria were the following: (I) the burn site in the head, face, and neck did not reach the cutoff of $10 \%$ of the wound area; (II) the burn wound was severely infected or contaminated; (III) shock had not been effectively treated; (IV) there was a serious underlying disease or combined injury; and (V) the patient did not pass the anesthesia assessment or was otherwise unable to meet the surgical requirements. All procedures performed in this study involving human participants were in accordance with the Declaration of Helsinki (as revised in 2013). This study was approved by the Ethics Committee of the PLA General Hospital (approval number: 2021KY032KS001) and informed consent was taken from all the patients' guardians.

\section{Study grouping and treatment}

Of the 78 children, 37 children received PPSR (PPSR group), with an average age of $1.59 \pm 1.18$ years, 22 of whom were male and 15 female. The burn area was $16.30 \% \pm 5.37 \%$. Children in the PPSR group were given PPSR combined with acellular xenogeneic pig skin covering after scab removal. Additionally, 41 children received routine dressing change (RDC group), with an average age of $1.74 \pm 1.39$ years, including 22 males and 19 females. The RDC group was treated with traditional debridement and dressing change. The wound dressing was changed with 
epidermal growth factor, silver-zinc antibacterial cream, and dressing change. The frequency of dressing change was adjusted once per day or once every other day depending on the condition of the wound.

\section{Equipment and materials}

Electric dermatome was acquired from Zimmer Biomet Holdings, Inc. (Warsaw, IN, USA). Heterogeneous acellular dermal matrix dressing: the heterogeneous acellular dermal matrix dressing was acquired from Jiangsu Youchuang Biomedical Technology Co., Ltd. (model DC-ADM-b, National Food and Drug Administration Standard 2014 No. 3641173; Qidong City, Jiangsu Province, China).

\section{Treatment}

The children were first given simple wound treatment in the outpatient and emergency department. The wound was disinfected with iodophor, with an attempt made to preserve the rot on the wound, and only the unreducible fold rot or severely contaminated rot was removed. Iodophor was again applied after simple debridement and disinfection. The children's wounds were covered with a single layer of petroleum jelly gauze and bandaged with sterile gauze, and the children were then admitted to hospital for treatment.

After admission, the children were given routine antishock and anti-infection treatment, nutritional support, and other treatments. Deep second-degree burn refers to heat damage including the dermis below the nipple layer, but part of the dermis still remains. The standard of appearance of deep second-degree wounds is: local burns are swollen, the epidermis is white or brownish-yellow, and occasionally there are smaller blisters. After the necrotic epidermis is removed, the wound is slightly wet, reddish or white with reddish, with tough texture, dull sensation, lower temperature, and red dots the size of miliary grains, or small branch-like blood vessels can be seen.

Children in the PPSR group received general anesthesia. After the anesthesia was stabilized, the wound surface was removed, which was followed by routine iodophor disinfection and application of a sterile sheet. If it was a limb wound, the surgical limb was lifted for 2 to 3 minutes, and regardless of the wound area, there was no need to inject water under the scab. An electric skin knife (Zimmer) was used at a $45^{\circ}$ angle to the cut surface of the wound. The thickness of the skin knife was adjusted to $0.1 \mathrm{~mm}$, and the width of the knife head could be selected from different sizes of cards depending on the scab. When scab was being cut, the electric dermatome was advanced forward carefully, and the angle between the dermatome and the wound plane was maintained. After the scab was cut, the exposed wound surface consisted of porcelain-white dermis, and there was still a small amount of necrotic tissue at the base of the wound. If there were large bleeding spots or some yellow fat particles on the wound surface after the scab was cut, the depth of the scab was considered to be too deep, and the force of the scab was adjusted or the angle of the electric knife was reduced. After the scab was cut, the wound was quickly covered with adrenaline saline gauze and pressed for 3-5 minutes to stop bleeding. After the scabs were cut, they were rinsed with $1.5 \%$ hydrogen peroxide solution and gentamicin saline solution, and then covered with acellular xenogeneic dermis (Jiangsu Youchuang). Sterile gauze was then applied externally with pressure according to the location, and then bandage and fixed. After the operation, an ordinary bed or a suspended bed was chosen according to the burn site to avoid pressure on the surgical site. If the whole body condition of the child was stable and the dressing had no penetration, the first dressing change time was made on the seventh day after the operation. The dressing change only replaced the excipients, and the heterogeneous dermis was not removed. The dressing was changed a day after the first dressing change, during which the separated part of the heterogeneous dermis and the base was found, and then cut off with tissue scissors. After the xenogeneic dermis was completely separated, if there was still a wound, the conventional iodophor/silver-zinc antibacterial cream + epidermal growth factor dressing was applied. If the formation of granulation tissue appeared and proved difficult to heal, skin graft surgery could be performed later.

The children in the RDC group were given conventional wound debridement and dressing. According to the condition of wound secretions, iodophor disinfection, normal saline to clean the wound, or silver-zinc antibacterial cream + epidermal cell growth factor for wound dressing were used. The frequency of dressing change was every other day until the wound had healed. If granulation tissue formed on the wound and proved difficult to heal, skin grafting could be performed later.

\section{Research indicators}

Research indicators examined included children's gender, age, burn area, hospital stay, wound-healing time (unhealed 
Table 1 Comparison of general clinical data of the burn patients

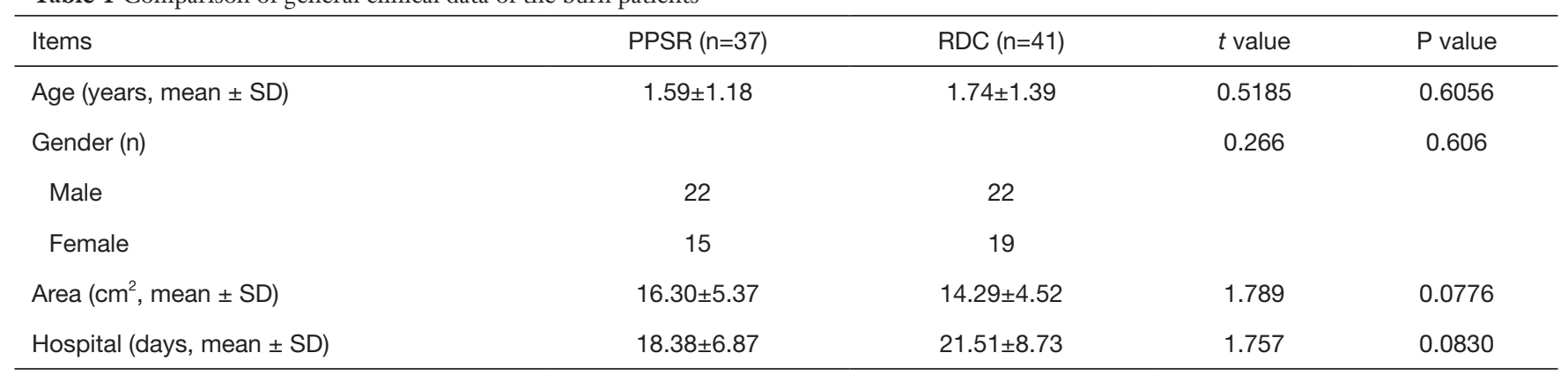

PPSR, precise partial scab removal; RDC, routine dressing change; SD, standard deviation.

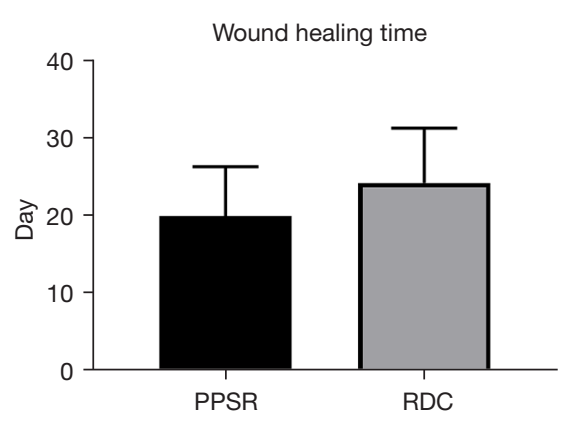

Figure 1 Wound-healing time. PPSR, precise partial scab removal; RDC, routine dressing change.

wound $\leq 1 \%$ ), duration of fever (highest body temperature of the day $\geq 38.5^{\circ} \mathrm{C}$ ), antibiotic use time, number of postoperations (excluding some scab removal surgery), and the total cost of hospitalization. During the operation, the removed crust was taken for pathological examination, and the depth of the removal was observed.

\section{Statistical analysis}

Statistical analysis was performed using SPSS22.0 statistical software (IBM Corporation, Armonk, NY, USA). Continuous data are expressed as mean and standard deviation. Comparisons between groups were conducted using the $t$-test, and a $\mathrm{P}$ value $<0.05$ was considered statistically significant.

\section{Results}

\section{Baseline characteristics}

There were no significant differences in age, gender, or burn area between the RDC group and the PPSR group $(\mathrm{P}>0.05)$, indicating that the two sets of data were comparable. The difference in hospitalization time between the two groups was also not statistically significant (Table 1).

\section{Wound-bealing time}

The wound-healing time of the PPSR group was 19.86 \pm 6.4 days, and the wound-healing time of the RDC group was $24.15 \pm 7.12$ days, which was a statistically significant difference ( $\mathrm{P}=0.0068$; Figure 1).

\section{The duration of fever and the time of antibiotic use}

The duration of fever in the PPSR group was $2.62 \pm$ 1.99 days, and the duration of fever in the RDC group was $4.44 \pm 3.10$ days $(\mathrm{P}=0.0032$; Figure 2$)$. The antibiotic use time of the PPSR group was $4.0 \pm 1.33$ days, and the antibiotic use time of the RDC group was $4.83 \pm 1.88$ days $(\mathrm{P}=0.0292$; Figure 2).

\section{Overall hospitalization cost}

The average overall hospitalization cost of the PPSR group was yuan renminbi $¥ 37,852.84 \pm 10,894.64$, and the average overall hospitalization cost of the RDC group was yuan renminbi $¥ 38,047.46 \pm 19,450.37(\mathrm{P}=0.9573)$.

\section{Histopathology}

The thickness of the scab removed in PPSR group was about $0.15 \mathrm{~mm}$, and the appearance changed to a thin leather-like texture, which was stained dark brown due to iodophor disinfection (Figure 3A). Pathological examination of the removed crust showed that the crust included the epidermis and the superficial dermis; the boundary between 

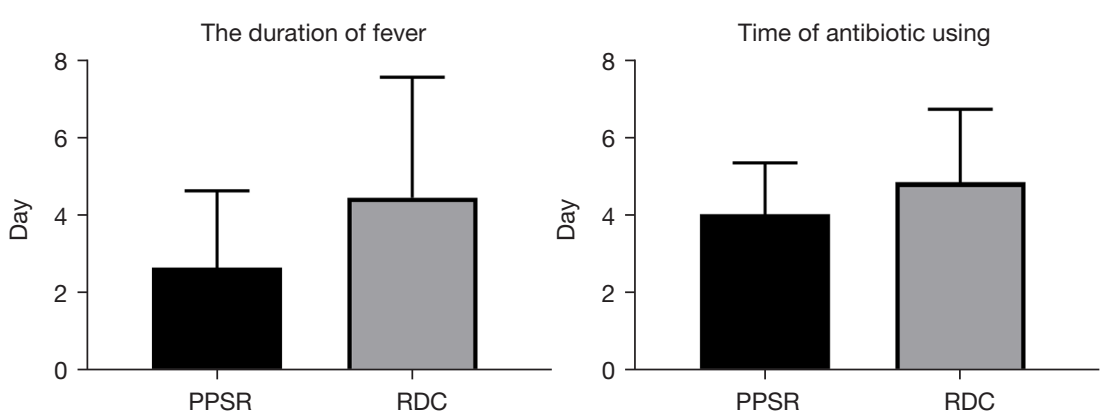

Figure 2 The duration of fever and time of antibiotic using. PPSR, precise partial scab removal; RDC, routine dressing change.
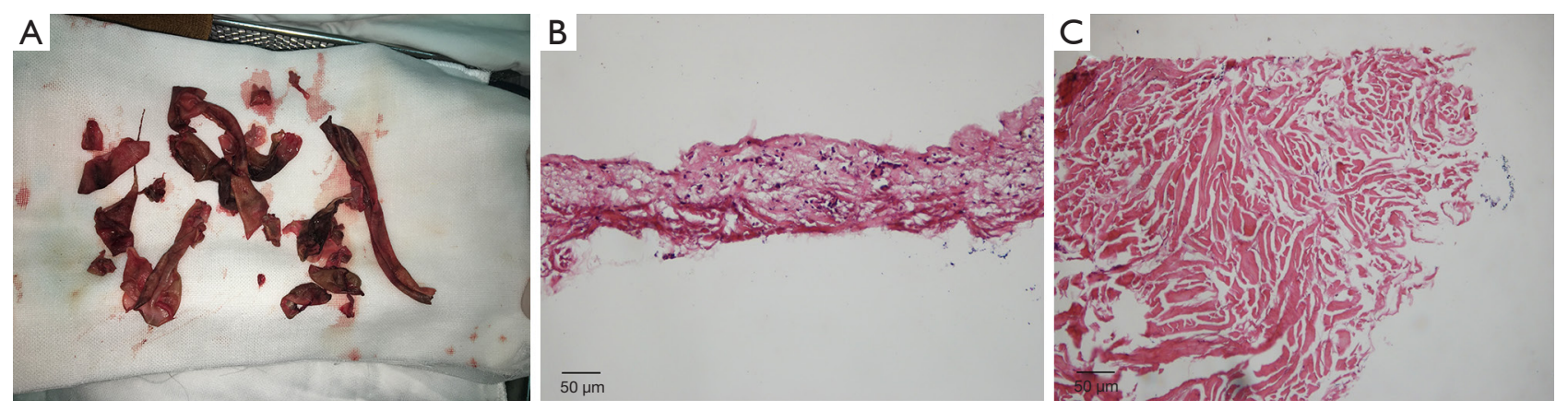

Figure 3 Pathological findings of scab. (A) A scab removed by the electric dermatome; (B) pathological examination of the scab after the scab was cut; (C) basal pathological examination of the wound after precise scab removal. (B,C) Hematoxylin and eosin staining; magnification: $\times 200$.

the epidermis and the dermis was not clear; the epidermis and the dermis showed cell necrosis; the nucleus had shrunk and collapsed, showing briquette-like changes; the collagen fibers had coagulated and fused, causing an increase in the gap between them (Figure 3B). After the scab was cut, pathological examination of the base of the wound was performed. Some dermal cells were still seen to be necrotic, and deep collagen fibers were coagulated and fused, but no fat tissue was exposed (Figure 3C).

\section{Typical case}

A male child, 1 year and 2 months old, had 30\% total body surface area (TBSA) deep second-degree burns to his trunk and limbs. Two hours after the injury, he came to the emergency department and was admitted to the hospital. Two days after the injury, the wounds of the trunk, left upper limb, and left lower limb were accurately cut and covered with pig acellular dermis. The dressing was changed for the first time 7 days after the operation. The pig acellular dermis covering the wound was clean and odorless, and there was no obvious exudation or secretion. The sterile gauze continued to be applied; 15 days after the operation, the pig acellular dermis had completely fallen off. Most of the wounds had healed, and 22 days after the injury, the wounds had healed completely. Follow-up for 6 months after operation showed that some scars were hyperplastic, as shown in Figure 4.

\section{Discussion}

The treatment of burns and scalds in children remains a difficult issue for clinicians in burn surgery. Children, especially those who have just acquired the ability to walk at the age of 1-2 years, are often the most vulnerable to accidental burns due to their curiosity for the world and their inability to avoid its dangers. From the analysis of the cause of injury, hydrothermal scalds account for the largest proportion of burns, with more boys than girls being affected (9). Due to the developmental characteristics of the skin of young children, wounds caused by hot fluids may often cause deep second-degree or deeper wounds, which 

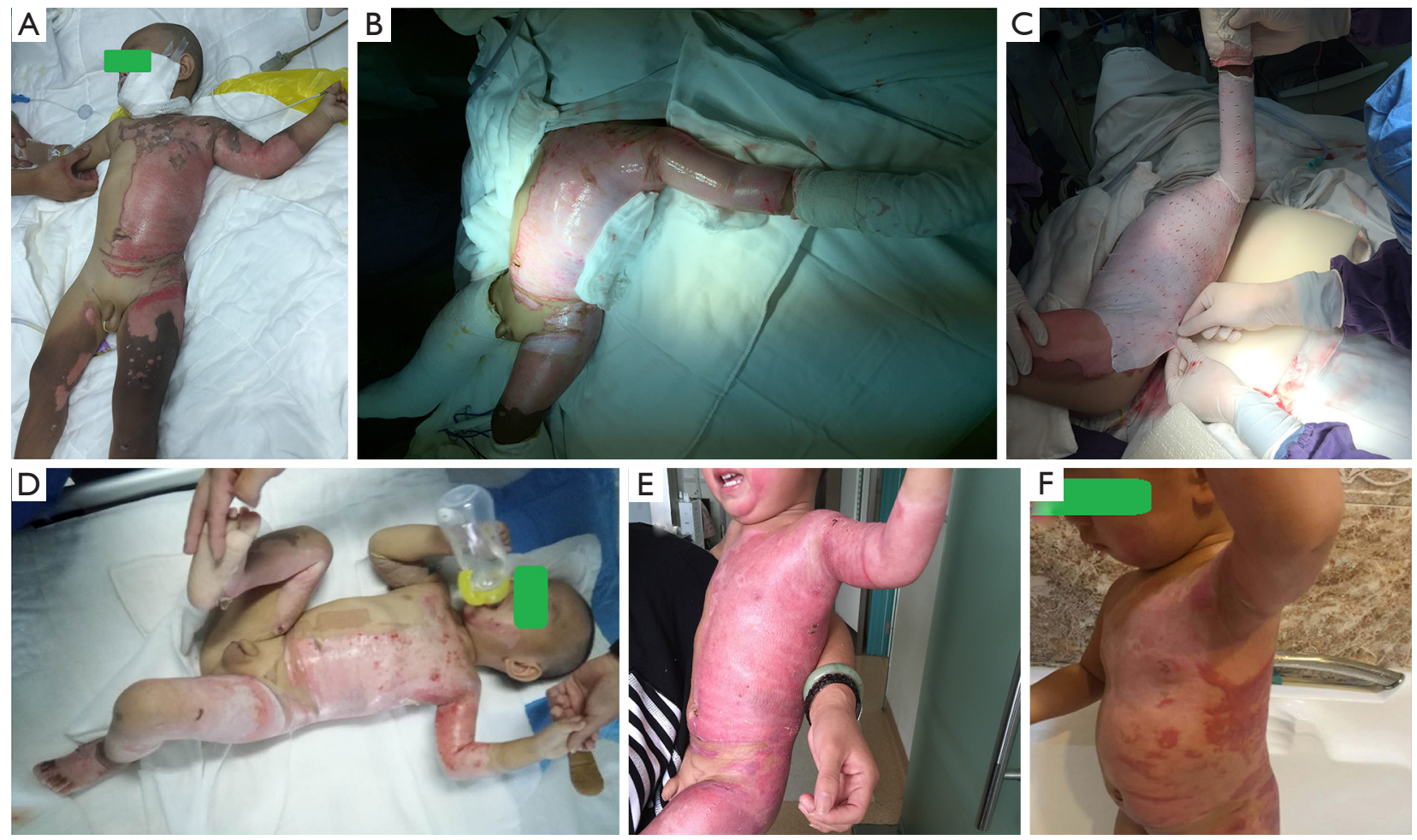

Figure 4 A typical case (boy). (A) Wound condition before admission to the hospital for debridement and bandaging; (B) wound base condition after scab removal during precision scab removal; $(\mathrm{C})$ status after porcine acellular dermis covering during precision scab removal; (D) 15 days after operation, the pig acellular dermis was completely shed; (E) wound healing at 22 days after injury; (F) follow-up at 6 months after injury. This group of pictures was published with the consent of the patient's guardian.

then require a longer healing time, resulting in more serious hypertrophic scars (10). Hypertrophic scars on joints and moving parts may cause dysfunction to varying degrees, which will seriously affect the growth and development of children and their quality of life (11). In addition, children may suffer traumatic emergency syndrome and possible psychological problems after burns and scalds (12). The psychosocial rehabilitation of burned hospitalized children depends largely on the family members, close friends, and burn staff (13). Some research indicates that children's accidental burns and scalds can also affect the mental health of their parents (14). In addition, the economic burden of children's burns and scalds on the family and society cannot be ignored (15). Indeed, finding a more appropriate and effective treatment plan for children with deep seconddegree burns is an important problem faced by burn surgery clinicians.

The current clinical treatment strategies for treating children's burns and scalds can be divided into two main approaches: nonsurgical and surgical. Nonsurgical methods include traditional wound dressing and the closed negative pressure suction method. For children, the pain of changing dressings for burns often leads to poor compliance of children with dressing changes for burn wounds, which not only increases the possibility of psychological problems in children and their families, but also affects the effect of dressing changes (16). The closed negative pressure suction method has higher requirements on the wound site, the compliance of the child, and on the finances of the family. If surgery is adopted, the choice of surgical method is crucial. Different methods of debridement and coverage of deep second-degree wounds will undoubtedly lead to different treatment results. The advantages and disadvantages of traditional scalpel debridement and roller knife debridement are very obvious. In recent years, there have been reports regarding the application of scab grinding in the debridement of deep second-degree wounds. The first report on the application of scab grinding in the field of debridement was first seen in 1977 (17). Since then, its application range has also been expanded. In 2006, the early 
application of grinding scabs for debridement in children with burns was reported (18). There are also differences in the selection of these surgical instruments for scab grinding. Sandpaper, grinding wheels, and steel wire balls are all used for scab grinding, and the results obtained are not easy to quantify under the same standard (19). The application of electric dermatome has made up for this shortcoming to a certain extent. The electric dermatome was originally used for the removal of healthy skin, and its skin thickness adjustment has proven to be relatively accurate. However, we found that for deep second-degree wounds, the electric dermatome knife can provide a relatively accurate debridement effect. Of course, although we can adjust the thickness of the scab by adjusting the scale of the electric dermatome, the actual thickness of the scab is still affected by many factors, for example, the strength of the surgeon holding the electric dermatome, the angle formed by the electric dermatome and the wound, the location of the wound, and the degree to which the assistant fixes the wound tissue. The degree of precise control of the scab offered by the electric dermatome is still significantly better than that provided by other methods.

Regardless of the debridement method chosen, the choice of covering method after debridement is also an important factor in determining healing. The methods of removing autologous skin covering, applying various excipients, or covering with biological materials have been reported. Different materials, such as hyaluronic acid dressing, chitosan, and nano-silver, each have their own advantages $(20,21)$. In recent years, the application of a variety of biological materials, such as platelet-rich plasma and acellular xenogeneic skin, has also achieved satisfactory results (22). Based on the characteristics of children with burns and scalds, we apply acellular xenogeneic (pig) dermal matrix to cover the deep second-degree wounds after precise scabs. There are many benefits to this method, including the reduction of the number of dressing changes, the prolongation of the first dressing change after the operation, the longer duration of protective effect on the wound, and the lower the incidence of infection and improvement in body temperature control. In the dressing change 7 days after the operation, only the auxiliary materials of the outer layer of the acellular xenogeneic dermis were replaced. There was basically no pain during the replacement, and the dermis gradually fell off without pain until the wound healed under the acellular dermis. This method greatly reduces the pain of dressing change, reduces the psychological impact of dressing change on children and parents, and decreases medical resources and costs.

The results showed that there was no statistically significant difference in hospitalization time between the RDC and PPSR groups, but the wound-healing time of the PPSR group was significantly shorter than that of the RDC group. We believe the reason for this is that, in the late stage of wound healing of the RDC group, some parents chose an outpatient dressing change when the unhealed wound area was small, thus leading to a significantly shorter hospital stay than the wound-healing time. In addition, in the PPSR group, a large amount of necrotic skin tissue was removed through scab removal surgery, which significantly reduced the burden of infection on the wounds of the children. Consequently, the duration of fever was significantly shorter than that of the RDC group. The shortening of fever time can also help clinicians avoid use of antibiotics. The overall cost of treatment in the PPSR group was lower than that of the RDC group, but the difference was not statistically significant. We assume that because the unit cost of the pig acellular dermis used in our clinic is still relatively expensive and the precision scab surgery involves additional costs for anesthesia, the cost savings associated with PPSR are still small.

The electric dermatome was used to accurately cut the scabs of children with deep second-degree burns, and the method of concurrent acellular xenogeneic dermal covering achieved an ideal therapeutic effect in this study. This method has played an active role in shortening wound healing time, reducing the frequency of dressing changes, shortening the time of fever in children, lowering the frequency of antibiotic use, lowering the frequency of wound dressing changes, and reducing the pain of children and the burden on parents. With the increase in the number of cases and the further refined control of the thickness of the scab, it is believed that there is room for further improvement in the treatment effect. When surgeons need to choose treatment for children with DPBWs, this method is worth considering and promoting.

This study also has certain limitations. Because the children came from a variety of places, this study did not collect the follow-up data of each child completely. In the future, we will contact the guardian of the children using several channels and collect follow-up data for further analysis.

\section{Acknowledgments}

Funding: This study was supported by PLA General Hospital 
Big Data Project "The establishment of emergency clinical database for burn and study of scar prediction model" (2019MBD-054) and Beijing Municipal Commission of Science and Technology (Z181100001718037).

\section{Footnote}

Reporting Checklist: The authors have completed the STROBE reporting checklist. Available at https://dx.doi. org/10.21037/tp-21-500

Data Sharing Statement: Available at https://dx.doi. org/10.21037/tp-21-500

Conflicts of Interest: All authors have completed the ICMJE uniform disclosure form (available at https://dx.doi. org/10.21037/tp-21-500). The authors have no conflicts of interest to declare.

Ethical Statement: The authors are accountable for all aspects of the work in ensuring that questions related to the accuracy or integrity of any part of the work are appropriately investigated and resolved. All procedures performed in this study involving human participants were in accordance with the Declaration of Helsinki (as revised in 2013). This study was approved by the Ethics Committee of the PLA General Hospital (approval number: 2021KY032KS001) and informed consent was taken from all the patients' guardians.

Open Access Statement: This is an Open Access article distributed in accordance with the Creative Commons Attribution-NonCommercial-NoDerivs 4.0 International License (CC BY-NC-ND 4.0), which permits the noncommercial replication and distribution of the article with the strict proviso that no changes or edits are made and the original work is properly cited (including links to both the formal publication through the relevant DOI and the license). See: https://creativecommons.org/licenses/by-nc-nd/4.0/.

\section{References}

1. Kaźmierski M, Mańkowski P, Jankowski A, et al. Comparison of the results of operative and conservative treatment of deep dermal partial-thickness scalds in children. Eur J Pediatr Surg 2007;17:354-61.

2. Jeschke MG, Herndon DN. Burns in children: standard and new treatments. Lancet 2014;383:1168-78.
3. Blome-Eberwein SA, Amani H, Lozano DD, et al. A biodegradable synthetic membrane to treat superficial and deep second degree burn wounds in adults and children - 4 year experience. Burns 2021;47:838-46.

4. Finlay V, Burrows S, Burmaz M, et al. Increased burn healing time is associated with higher Vancouver Scar Scale score. Scars Burn Heal 2017;3:2059513117696324.

5. Yontar Y, Coruh A, Dinc N, et al. Steel Wool-Aided Dermabrasion of Deep Partial-Thickness Burns. J Burn Care Res 2017;38:179-86.

6. Amani H, Dougherty WR, Blome-Eberwein S. Use of Transcyte and dermabrasion to treat burns reduces length of stay in burns of all size and etiology. Burns 2006;32:828-32.

7. Guo ZQ, Qiu L, Gao Y, et al. Use of porcine acellular dermal matrix following early dermabrasion reduces length of stay in extensive deep dermal burns. Burns 2016;42:598-604.

8. Li F, Chi YF, Hu Q, et al. Effects of minimally invasive tangential excision in treating deep partial-thickness burn wounds on trunk and limbs in pediatric patients in the early stage post burn. Zhonghua Shao Shang Za Zhi 2018;34:714-8.

9. Nguyen NL, Ngo MD. Profile and outcome of burn injuries amongst preschool children in a developing country. Ann Burns Fire Disasters 2019;32:267-71.

10. Karlsson M, Steinvall I, Sjöberg F, et al. Burn scar outcome at six and 12 months after injury in children with partial thickness scalds: Effects of dressing treatment. Burns 2020;46:546-51.

11. Radwan NL, Ibrahim MM, Mahmoud WS. Effect of Wiihabilitation on spatiotemporal parameters and upper limb function post-burn in children. Burns 2021;47:828-37.

12. Price J, Kassam-Adams N, Alderfer MA, et al. Systematic Review: A Reevaluation and Update of the Integrative (Trajectory) Model of Pediatric Medical Traumatic Stress. J Pediatr Psychol 2016;41:86-97.

13. Van Niekerk A, Jacobs R, Hornsby N, et al. Enablers of psychosocial recovery in pediatric burns: perspectives from the children, parents and burn recovery support staff. BMC Pediatr 2020;20:289.

14. Lernevall LST, Moi AL, Cleary M, et al. Support needs of parents of hospitalised children with a burn injury: An integrative review. Burns 2020;46:771-81.

15. Beaulieu E, Zheng A, Rajabali F, et al. The Economics of Burn Injuries Among Children Aged 0 to 4 Years in British Columbia. J Burn Care Res 2021;42:499-504.

16. Yildirim M, Koroglu E, Yucel C, et al. The effect of 
hospital clown nurse on children's compliance to burn dressing change. Burns 2019;45:190-8.

17. Krant SM, Arons MS. Dermabrasion debridement of the deep dermal burn. Plast Reconstr Surg 1977;60:68-73.

18. Esposito G, Gravante G, Montone A. Use of early dermabrasion in pediatric burn patients. Plast Reconstr Surg 2006;118:573-5; author reply 575.

19. De Souza BA, Furniss D, Jawad M. The use of sandpaper as a precision tool for dermabrasion in burn surgery. Plast Reconstr Surg 2005;116:350-1.

20. Esposito G, Gravante G, Filingeri V, et al. Use of hyaluronan dressings following dermabrasion avoids

Cite this article as: Chi Y, Yin H, Chen X, Hu Q, Liu W, Feng L, Chai J. Effect of precise partial scab removal on the repair of deep partial-thickness burn wounds in children: a retrospective study. Transl Pediatr 2021;10(11):3014-3022. doi: 10.21037/tp21-500 escharectomy and facilitates healing in pediatric burn patients. Plast Reconstr Surg 2007;119:2346-7.

21. Jyoti K, Malik G, Chaudhary M, et al. Chitosan and phospholipid assisted topical fusidic acid drug delivery in burn wound: Strategies to conquer pharmaceutical and clinical challenges, opportunities and future panorama. Int J Biol Macromol 2020;161:325-35.

22. Farjah MH, Farahpour MR. Efficacy of topical plateletrich plasma and chitosan co-administration on Candida albicans-infected partial thickness burn wound healing. Burns 2020;46:1889-95. 